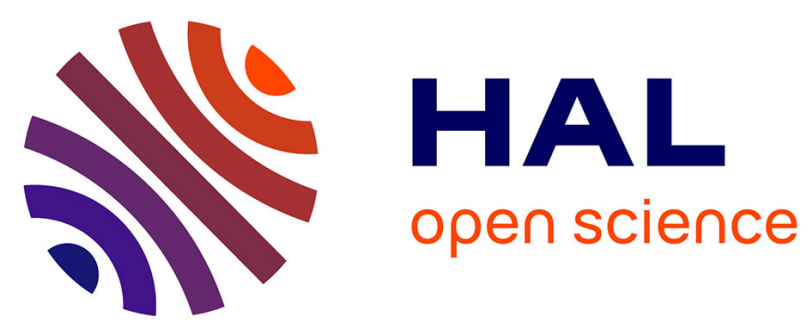

\title{
Current Distribution Identification in Fuel Cell Stacks From External Magnetic Field Measurements
}

Mathieu Le Ny, Olivier Chadebec, Gilles Cauffet, Jean-Marc Dedulle, Yann Bultel, Sébastien Rosini, Yannick Fourneron, Patrick Kuo-Peng

\section{- To cite this version:}

Mathieu Le Ny, Olivier Chadebec, Gilles Cauffet, Jean-Marc Dedulle, Yann Bultel, et al.. Current Distribution Identification in Fuel Cell Stacks From External Magnetic Field Measurements. IEEE Transactions on Magnetics, 2013, 49 (5), pp.1925-1928. 10.1109/TMAG.2013.2239967 . hal00822690

\section{HAL Id: hal-00822690 https://hal.science/hal-00822690}

Submitted on 27 Jan 2021

HAL is a multi-disciplinary open access archive for the deposit and dissemination of scientific research documents, whether they are published or not. The documents may come from teaching and research institutions in France or abroad, or from public or private research centers.
L'archive ouverte pluridisciplinaire HAL, est destinée au dépôt et à la diffusion de documents scientifiques de niveau recherche, publiés ou non, émanant des établissements d'enseignement et de recherche français ou étrangers, des laboratoires publics ou privés. 


\title{
Current Distribution Identification in Fuel Cell Stacks From External Magnetic Field Measurements
}

\author{
Mathieu Le Ny ${ }^{1,2}$, Olivier Chadebec ${ }^{1,5}$, Gilles Cauffet ${ }^{1}$, Jean-Marc Dedulle ${ }^{3}$, Yann Bultel ${ }^{2}$, Sébastien Rosini ${ }^{4}$, \\ Yannick Fourneron ${ }^{4}$, and Patrick Kuo-Peng ${ }^{5}$ \\ ${ }^{1}$ G2ELab (UMR CNRS 5269, Grenoble INP, UdS, UJF), University of Grenoble, Grenoble, 38041, France \\ ${ }^{2}$ LEPMI (UMR CNRS 5279, Grenoble INP, UdS, UJF), University of Grenoble, Grenoble, France \\ ${ }^{5}$ GRUCAD Departamento de Engenharia Elétrica - CTC, Florianópolis, Santa Catarina, CEP 88040-970, Brasil \\ ${ }^{3}$ Laboratoire des Matériaux et du Génie Physique Minatec, University of Grenoble, Grenoble, 38016, France \\ ${ }^{4}$ Commissariat a l'Energie Atomique (CEA/LITEN),LITEN/DTH/LIGE, Grenoble cedex 9, 38054, France
}

\begin{abstract}
This paper presents a new non invasive technique for fuel cell diagnosis. The method relies on the measurements of the magnetic field signature generated by a working fuel cell. Knowing the relationship between currents and magnetic field, it is possible to estimate the current density by solving an inverse problem. This problem being ill-posed, original current and sensors basis are proposed to improve the reconstruction process. The experimental set-up remains simple, based on few fixed sensors and shows a good efficiency.
\end{abstract}

Index Terms-Current density identification, fuel cell, inverse problems, magnetostatics.

\section{INTRODUCTION}

$\mathbf{T}$ HIS paper proposes an innovative non-intrusive diagnostic method for fuel cell stacks that provides a significant improvement in terms of estimation quality in comparison with existing non-invasive tools. The technique proposed here is to measure the magnetic field around a fuel cell in operation. Knowing the relationship between currents and this field, it becomes possible to evaluate the internal current density by solving an inverse problem. This technique is already well known and has provided many interesting results in different research fields. For instance, let us mention the reconstruction of currents density in circuit breakers [1], in bus-bars [2] and even in the human heart [3].

In this work, we focus on the application of this technique to fuel cell stack. Fuel cells are energy conversion devices that produce electricity directly from hydrogen and oxygen. A stack is an assembly of elementary cells that allows the creation of devices with high power. Currently, in spite of recent undeniable progresses, these devices present certain drawbacks and improvements must be made in order to use them in real industrial application.

Fuel cells are prone to chemical, mechanical, or thermal hard constraints that inevitably lead to their degradation. These phenomenons are still not very well understood but non-uniform fuel/air flows, hot spots and non uniformity of materials within the stack can be the reasons of such degradations. In order to better understand the real behavior of stacks, the knowledge of local current density flowing within the system is fundamental. If the global value of the current is obvious to measure (with a simple ammeter), getting its local densities is not so easy. If some intrusive technique exist [4], their drawback is that the internal presence of sensors in the stack disturbs its operation mode. Then it is hard to discriminate if the faulty mode is due to the stack itself or to the diagnosis set-up.
Non-intrusive approaches based on the measurement of the external magnetic field and the resolution of an inverse problem in order to identify internal current density has already been proposed in [5], [6]. In these works, a tri-axial magnetic sensor is moved in the 3D space around the stack with a robot in order to characterize the magnetic signature. Several hundred of measurements are made and the acquisition time is about fifteen minutes. Such system is extremely complex to develop and can only be used in a laboratory context.

The originality of the approach presented here is that it requires a very small number of magnetic field measurements (thirty against several hundred for what has been done so far). The instrumentation is facilitated and the use of a robot moving a sensor is no longer necessary. Thirty sensors are fixed around the stack and enable an instantaneous measurement (against at least fifteen minutes with a robot). The major advantage is that the internal state of the stack does not vary during the measurement time. This low number of sensors is possible thanks to assumptions on the current distribution and to an adequate choice of positioning and orientation. These choices and assumptions are explained in the second part of this article. The third part will present the validation of the method on a stack simulator. Then, experimental results on a real stack are presented.

\section{Methodology}

\section{A. Forward Modeling}

Before solving an inverse problem, we have to carefully study the forward one, i.e., from a given current distribution, how to compute the magnetic field outside. We consider that the stack and its environment are free of any ferromagnetic pieces and that no disturbing sources create additional magnetic field. Therefore, the relation between currents and field can be simply expressed by the Biot-Savart operator.

$$
\begin{aligned}
\mathbf{B}(\mathbf{r}) & =\int_{\Omega_{\mathrm{S}}} \mathbf{j}\left(\mathbf{r}_{\mathbf{S}}\right) \wedge \mathbf{F}\left(\mathbf{r}, \mathbf{r}_{\mathbf{S}}\right) d \Omega_{S} \\
\mathbf{F}\left(\mathbf{r}, \mathbf{r}_{\mathbf{S}}\right) & =\frac{\mu_{0}}{4 \pi} \frac{\mathbf{r}-\mathbf{r}_{\mathbf{S}}}{\left|\mathbf{r}-\mathbf{r}_{\mathbf{S}}\right|^{3}}
\end{aligned}
$$


where $\mathbf{B}$ is the induction at point $\mathbf{r}, \mathbf{j}$ is the current density at the integration point $\mathbf{r}_{\mathbf{s}}, \mu_{0}$ is the permeability of free space and $\Omega_{S}$ is the volume of the stack where current flows.

The (1) cannot be solved analytically and we have to discretize it in order to get the solution. Let us consider a parametization of current density thanks to $\mathrm{N}$ basis function $\boldsymbol{\Phi}_{\mathbf{p}}$.

$$
\mathbf{j}\left(\mathbf{r}_{\mathbf{S}}\right)=\sum_{\mathrm{p}=1}^{\mathrm{N}} \boldsymbol{\Phi}_{\mathrm{p}}\left(\mathbf{r}_{\mathbf{S}}\right) \mathbf{J}_{\mathrm{p}}
$$

For instance, if the space $\Omega_{\mathrm{s}}$ is meshed by several cells, each cell carries a single $\boldsymbol{\Phi}_{\mathrm{p}}$ function (0-order shape functions that are uniform on one cell and null elsewhere). This kind of the problem discretization seems maybe the most natural and is the most found in the literature [5]. We will see later that it is not the approach chosen in this work.

Let us now consider that the magnetic field is measured on a sensor number $k$ associated to a projection function $\psi_{\mathbf{k}}$.

$$
B_{k}=\int_{\Omega} \boldsymbol{\Psi}_{k}(\mathbf{r}) \cdot \mathbf{B}(\mathbf{r}) \mathrm{d} \Omega
$$

$\mathrm{B}_{\mathrm{k}}$ is the projection of the induction on a sensor function, i.e., a measured value and $\Omega$ is the whole 3D domain. For instance, if we consider a punctual directional sensor, $\boldsymbol{\psi}_{\mathbf{k}}$ is a Dirac function associated to a vector oriented in the same direction than the sensor.

Merging (1), (3) and (4), we get the discretized forward expression of our problem for $\mathrm{N}$ current basis and for the sensor number $k$.

$$
\begin{aligned}
\mathrm{B}_{\mathrm{k}} & =\sum_{\mathrm{p}=1}^{\mathrm{N}} \mathbf{S}_{\mathrm{kp}} \mathrm{J}_{\mathrm{p}} \\
\mathrm{S}_{\mathrm{kp}} & =\int_{\Omega} \boldsymbol{\Psi}_{\mathrm{k}}(\mathbf{r}) \cdot \int_{\Omega_{\mathrm{S}}} \boldsymbol{\Phi}_{\mathrm{p}}\left(\mathbf{r}_{\mathbf{S}}\right) \wedge \mathbf{F}\left(\mathbf{r}, \mathbf{r}_{\mathrm{S}}\right) \mathrm{d} \Omega_{\mathrm{S}} \mathrm{d} \Omega
\end{aligned}
$$

Let us notice that this relation is linear (as of course the BiotSavart law). With several sensors, we get a linear system linking the sources (characterized by $\mathrm{N}$ coefficients $\mathrm{J}_{\mathrm{p}}$ ) and the measured magnetic field (characterized by $M$ coefficients $B_{k}$ ).

$$
\mathbf{B}=\mathbf{S} \mathbf{J}
$$

where $\mathbf{B}$ is the measurements vector, $\mathbf{S}$ is a $\mathbf{M} \times \mathrm{N}$ matrix defined by (6) and $\mathbf{J}$ are the unknowns we want to determine.

\section{B. Inverse Modeling}

In order to reduce the number of degrees of freedom of the inverse problem and to improve the reconstruction process, we have to properly choose $\boldsymbol{\Phi}_{\mathbf{p}}$ and $\boldsymbol{\psi}_{\mathbf{k}}$ functions. The originality of this work is inherent to this choice. Once these functions are properly chosen, the problem remains ill-posed, i.e., the solution is not unique. We will have to regularize it in order to get the final solution. Let us notice that better the projection functions selection is, lighter the regularization process will be.

1) Choice of $\boldsymbol{\Phi}_{\boldsymbol{p}}$ (Current Basis): Two kinds of anomaly can arise in a fuel cell stack. A localized anomaly (flooding, hot spot) on membrane electrode assembly modifies the current only around the anomaly. More global faults (like wrong supply of gas, for instance) can also occur. For local anomaly, we can bet that it will not have an important influence on the external

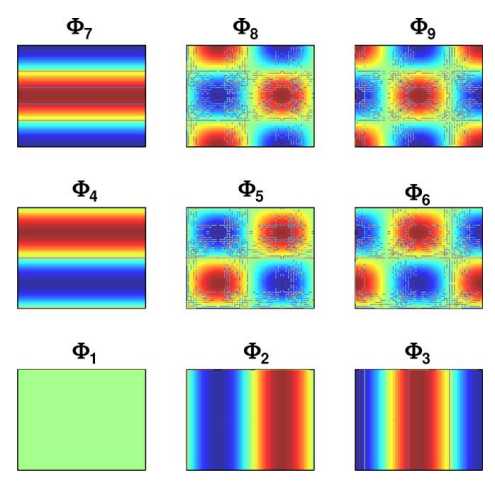

Fig. 1. First components of cross-section current density distributions used in order to generate $\boldsymbol{\Phi}_{\mathbf{p}}$ functions.

magnetic signature (they are generated by small current loops). So, we will not focus on the identification of such local inhomogeneous current densities. Global faults arising uniformly on all the cells of a stack will be search. This assumption (or better this reduction of the space search) reduces the potential number of unknowns. We consider that the current density in the active part of the stack is axial. This assumption does not suppose an infinite length for the fuel cell, the axial current density being limited to bipolar plates and membranes. We will see later that both collecting plates located at each extremity of the stack and in which flow non axial current will be properly taken into account.

Let us now define properly our $\boldsymbol{\Phi}_{\mathbf{p}}$ functions. In a first step, 2D Fourier transform basis are used to describe current density in fuel cell cross section. First components of this basis are represented below (see Fig. 1).

The $\boldsymbol{\Phi}_{\mathrm{p}}$ functions enabling the full representation of currents flows (including 3D effects due to current collection at each stack extremity) are generated by solving a steady state current conduction equation with a finite volume method. In this formulation, previous 2D current densities (Fig. 1) are introduced as a source term. Details of this formulation have been previously given by authors in [7]. Several current loops flowing inside the stack are generated and the associated field is computed thanks to the numerical integration of Biot-Savart law. An example of three $\boldsymbol{\Phi}_{\mathrm{p}}$ functions is represented on Fig. 2.

These functions can be discriminated in two categories. The first one is only composed of the $\boldsymbol{\Phi}_{1}$ function corresponding to a homogenous current distribution flowing through the stack (Fig. 2, left column). The second category is composed of all remaining functions (Fig. 2, central and right columns) for which current density is composed of internal loops flowing not through but inside the stack (the integral of current density on the cross-section being null by construction). Let us notice that the function $\boldsymbol{\Phi}_{1}$ represents the operation mode of the stack without any fault (maybe not exactly, but very nearby). Moreover, this common mode can easily be determined by the integral of the current flowing through the stack. This integral can be easily obtained thanks to a simple ammeter.

Other functions of the basis represent inhomogeneities i.e., faulty operation modes. We will only focus on the determination of these functions with the magnetic field measurements. Moreover, if we have a look to the $\boldsymbol{\Phi}_{\mathbf{2}}$ function, we see that it magnetically acts as a single current loop, i.e., not so far from 


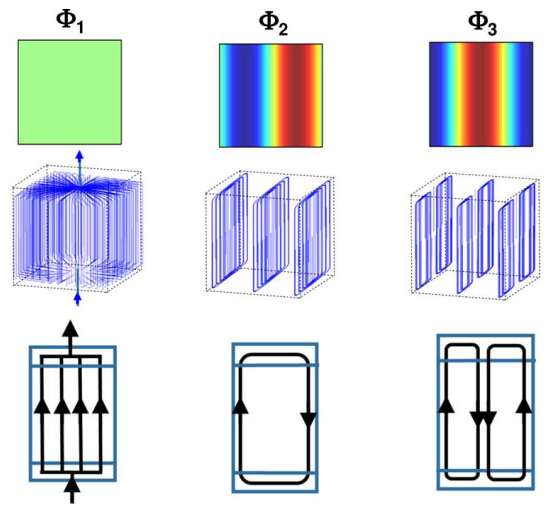

Fig. 2. Three examples of $\boldsymbol{\Phi}_{\mathbf{p}}$ functions. To the left, common current mode corresponding to the uniform current. Central column, the representation of a dipolar term and to the right a quadruple one. Up, 2D current distribution. Middle 3D representation and down schematic view.

a magnetic dipole. The function $\boldsymbol{\Phi}_{\mathbf{3}}$ is composed of two loops with opposite flowing directions, so it generates a magnetic field similar to which created by a quadrupole. Thus, our functions basis is not so different from a set of multipoles. This consideration can be very useful because a well-known result is that the field created by a multipole decreases with a $1 / \mathrm{r}^{\mathrm{n}+1}$ law where $\mathrm{n}$ is the order of the multipole $\left(1 / \mathrm{r}^{3}\right.$ for a dipole, $1 / \mathrm{r}^{4}$ for a quadrupole and so on). Thus we can conclude that it is useless to try to determine distribution of important orders because they are going to be naturally filtered by the distance between the stack and the sensor. So the decomposition can be truncated. In our application, we only keep the functions $\boldsymbol{\Phi}_{\mathbf{p}}$ of low spatial frequency. The number of unknowns for the inverse magnetic problem is then fixed to 48 .

2) Choice of $\boldsymbol{\psi} k$ (Sensors Basis): The magnetic field measurements are performed by using punctual magnetic sensors. Thus, we have to choose their locations and directions. In order to optimize the ratio between signal and noise, sensors are located as close as possible to the stack on a square path located on the middle section of the device. Sensors are located on a single plane.

Let us remember that the component associate to the first function $\left(\boldsymbol{\Phi}_{1}\right)$ can be easily and certainly more accurately determine by a direct measurement with an ammeter. Moreover, the magnetic field created by this function is far more important than the one created by the other functions $\boldsymbol{\Phi}_{\mathbf{p}}$. This field is in fact rather a problem than a potential source of information. Indeed, the useful information is the magnetic field created by inhomogeneities and certainly not the one created by the common mode that is much higher. This is a real difficulty for our problem because this huge field leads to the need of a high range of measurements keeping a very high accuracy. These two points are in contradiction. A good solution is to create a sensor network in which the common mode does not have any magnetic signature. If we have a look to the field created by $\boldsymbol{\Phi}_{1}$ (see Fig. 3, blue dotted arrows), we can see that this field is mainly orthoradial (exact directions can easily be computed). Our original solution is to use only single-axis sensors (represented with red solid arrow, Fig. 3) oriented in order to not measure the magnetic field generated by a homogeneous current flowing in the stack (quasi-radial orientation). So, the sensor array is sensitive only to inhomogeneous current (meaning defaults). This technique

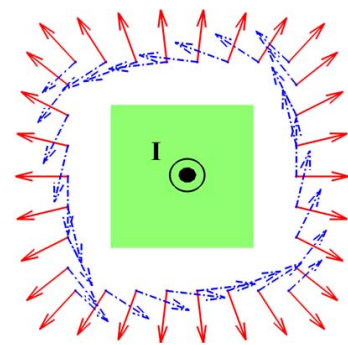

Fig. 3. Field created by the common current mode $\Phi_{1}$ (blue dotted arrows) and orientation of single-axis fluxgate magnetic sensors (red solid arrows).

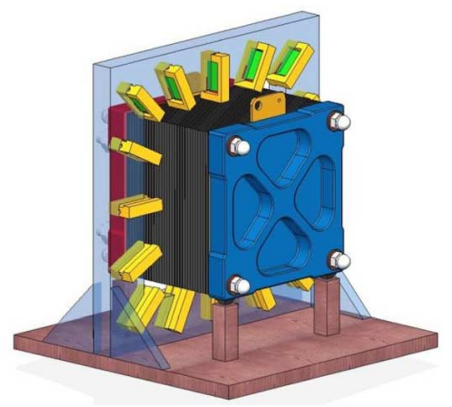

Fig. 4. CAD representation of the final measurement set: stack (blue, red and black) and sensors (yellow and green).

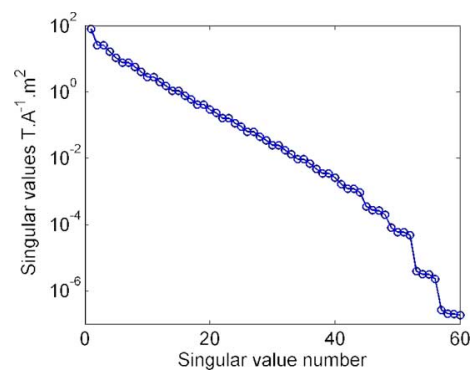

Fig. 5. Singular value decomposition of the forward model.

enables the use of accurate Fluxgate sensors with low range of measurement but excellent accuracy.

Let us notice that the final sensors positions on the square is obtained with an optimization procedure based on the Ds-optimality criterion [8] but optimized positions do not really differ from a regular distribution. This is why we do not focus on this step here. 30 sensors will be used. The final measurement set is presented in Fig. 4.

3) Final Resolution: Our inverse problem has been now properly parameterized (adequate choice of currents and magnetic bases). However, the singular value decomposition shows that the inverse problem remains ill-posed (see Fig. 5). Thus we have to regularize it. However, our previous choices considerably reduce the complexity of this step, the number of unknowns being considerably reduced. A truncated single value decomposition is thus used to get a stable solution [1]. Experiences (mainly realized on the stack simulator presented in next section) showed us that keeping the first fifteen singular value leads to good results.

\section{EXPERIMENTAL VALIDATION}

The approach has been first validated on a stack simulator in order to check the feasibility of the system (Fig. 6). This stack 

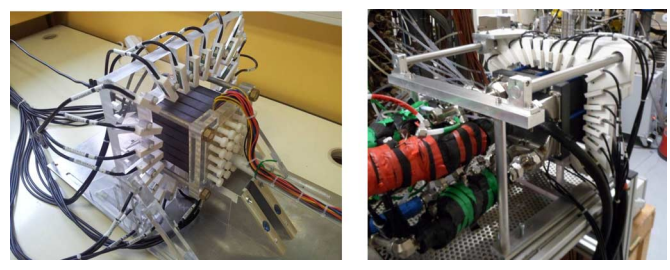

Fig. 6. Sensors network on the stack simulator (left) and on a real fuel cell stack (right).
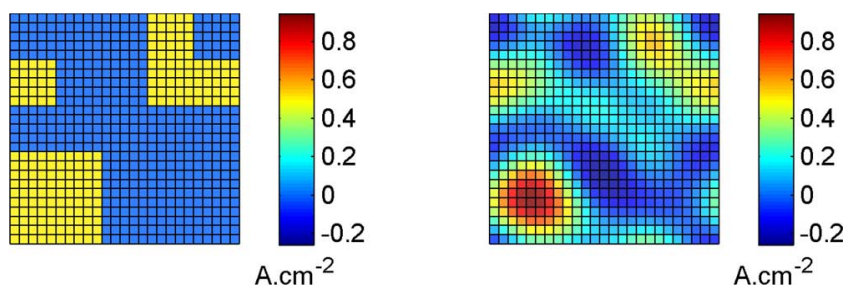

Fig. 7. Results obtained with the stack simulator (to the left profile of the current and to the right reconstructed distribution).

simulator is geometrically representative of a real stack and consists in 25 independent conductors in which we can impose a given current value. We can construct the $\boldsymbol{\Phi}$ basis quasi experimentally by feeding only one conductor; keeping the others without any current and then by measuring fields. The problem is solved and the obtained solution is compared to initial currents. Both distributions present a good agreement (see Fig. 7).

Our device has also been developed for a real stack. In this configuration, we face a real difficulty. Even if the system has been designed in theory to be not sensitive to current common mode, it cannot be exactly the case because some geometrical parameters are not precisely known, in particular the exact geometry of external currents feeds. Moreover, stacks environment is complex and leads to numerous mechanical constraints (see Fig. 5, right). The accurate positioning of sensors is then difficult and the current common mode creates on them a quite important residual magnetic field. A good way to proceed is then to work keeping the total current constant meaning to work with a differential approach. It is not always possible but both defaults proposed here lead to such configuration (oxygen starvation and artificial ageing of the stack during 60 hours). In order to compare obtained results with a reference, an internal current density measurement system is introduced inside the stack. Even if this intrusive tool disturbs certainly the operation mode, it enables to validate our approach. Once again, results obtained are satisfying (see Figs. 8-9), both distributions presenting a good agreement.

\section{CONCLUSION}

We have proposed a new and original magnetic tomography approach in order to reconstruct default in fuel cell stacks. It lies

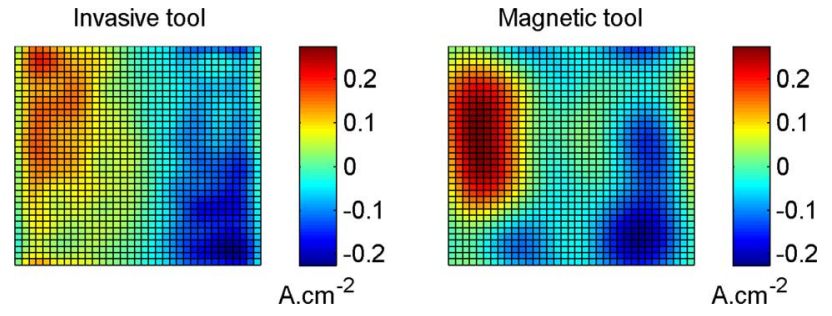

Fig. 8. Oxygen starvation of the stack: comparisons between currents density obtained with internal measurements (left) and with magnetic inverse problem (right).

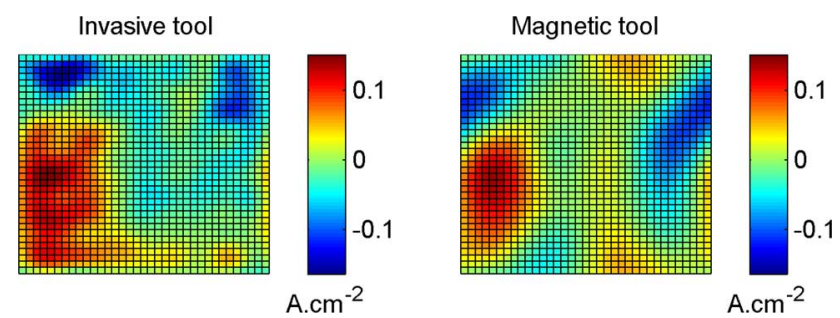

Fig. 9. Ageing attempt: comparisons between currents density obtained with internal measurements (left) and with magnetic inverse problem (right).

on the use of original current bases and of a sensors array configuration developed in order to be only sensitive to current inhomogeneity. Our approach is robust, enabling the use of accurate magnetic fluxgate sensor with a low range of measurements (less than $200 \mu \mathrm{T}$ ). The method has been validated on several test cases and has shown its potential.

\section{REFERENCES}

[1] L. Ghezzi, D. Piva, and L. Di Rienzo, "Current density reconstruction in vacuum arcs by inverting magnetic field data," IEEE Trans. Magn., vol. 48, no. 8, pp. 2324-2333, 2012.

[2] L. Di Rienzo, R. Bazzocchi, and R. Manara, "Circular arrays of magnetic sensors for current measurement," IEEE Trans. Instrum. Meas., vol. 50, no. 5, pp. 1093-1096, 2001.

[3] M. De Melis, K. Tanaka, and Y. Uchikawa, "Magnetocardiography signal reconstruction with reduced source space based on current source variance," IEEE Trans. Magn., vol. 46, no. 5, pp. 1203-1207, 2010.

[4] S. Sailler, S. Rosini, M. A. Chaib, J.-.Y. Voyant, Y. Bultel, F. Druart, and P. Ozil, "Electrical and thermal investigation of a self-breathing fuel cell," J. Appl. Electrochemistry, vol. 37, pp. 161-171, 2007.

[5] K.-.H. Hauer, R. Potthast, T. Wüster, and D. Stolten, "Magnetotomography-A new method for analysing fuel cell performance and quality," J. Power Sources, vol. 143, pp. 67-74, 2005.

[6] H. Lustfeld, M. Reißel, and B. Steffen, "Magnetotomography and electric currents in a fuel cell," Fuel Cells, vol. 4, pp. 474-481, 2009.

[7] M. Le Ny, O. Chadebec, G. Cauffet, J. M. Dedulle, and Y. Bultel, "A three dimensional electrical model of PEMFC stack," Fuel Cells, vol. 12, pp. 225-238, 2012, vol. 2.

[8] S. Begot, E. Voisin;, P. Hiebel, E. Artioukhine, and J.-M. Kauffmann, "D-optimal experimental design applied to a linear magnetostatic inverse problem," IEEE Trans. Magn., vol. 38, no. 2, pp. 1065-1068, 2002. 